\title{
GÉNERO Y EDUCACIÓN
}

De Pippi a Prisca

Por Silvia Tomasi

Maestra en Educación Infantil

Universidad de Cádiz - Facultad de Ciencias de la Educación

Grupo de Investigación HUM748

\section{Resumen}

En un marco actual en el que el discurso acerca de la igualdad de género ha cobrado fuerza e impulso, estimamos necesario abrir la reflexión acerca de las implicaciones que las políticas educativas vigentes han conllevado en el ámbito de la creación y promoción literaria. Reafirmando el valor de la literatura infantil y juvenil (desde ahora LIJ), como instrumento de desarrollo personal y construcción de la identidad y reconociendo que las ideologías políticas, culturales y sociales se extienden en los textos literarios para ofrecer una concreta visión del mundo, proponemos una rápida mirada acerca al panorama literario actual, en términos de producción y difusión. Frente a unas propuestas aun encorsetadas en tipificaciones sexuales $u$ otras excesivamente moralistas y artificiosas en su afán de revertir los estereotipos de género, algunos autores escriben para los niños con sinceridad sin más propósito que narrar desde su propio sentir. En este marco destacamos la figura de Bianca Pitzorno, una de las mayores representantes de la LIJ actual en Italia, inmejorable por su capacidad por representar identidades más allá del género y por traducir literariamente los problemas del ser mujer, chica o niña en la sociedad contemporánea.

\section{Abstract}

In a contemporary framework the discourse of gender has gained power and momentum, thus we estimate a necessity to give place to reflect about the implications that the current educational policies have led to in the range of creating and promoting literature. Reiterating the value of the YCL as a tool for the personal development and the construction of the identity, and acknowledging that the social, cultural and political ideologies are well spread in the literary texts offering a specific perspective of the world, we suggest a quick glance at the current literature panorama, in terms of production and broadcasting. Confronting proposals yet confined in sexual, moral and artificial classifications with the aim of reverting gender stereotypes, certain authors sincerely write children's literature without any purpose but to narrate from their own feelings. In this framework, we highlight the figure of Bianca Pitzorno, one of the greatest representatives of the current $\mathrm{YCL}$ in Italy, matchless for her 
ability to represent identities and for literally translating the problems of being a woman, a young girl or a child in the contemporary society.

\section{Palabras clave}

Género, Identidad, Literatura infantil y juvenil, Bianca Pitzorno.

\section{Keywords}

Gender, identity, Children and youth's literature, Bianca Pitzorno.

\section{La construcción de identidades más allá del género}

El discurso acerca del principio de igualdad entre hombres y mujeres y de la coeducación ha cobrado una especial fuerza en los últimos años, impulsándose desde las instituciones gobernativas unas políticas y actuaciones formativas que contribuyan a la sensibilización del alumnado y al desarrollo de actitudes y formas de pensar no sexistas. Partiendo de estas directivas en al ámbito educativo se han puesto en marcha, en mayor o menor medida, proyectos destinados a desarrollar actitudes de respeto y valoración de las diferencias ligadas al género.

Cabe señalar que las vivencias personales y familiares del niño son las que realmente ejercen el mayor impacto en la construcción de las relaciones humanas y de poder, de la identidad de género propia y de los demás y de las implicaciones derivadas de la pertenencia a uno u otro sexo, en términos de atribuciones y expectativas sociales.

Es precisamente por ello que el reto al que se enfrenta la educación es el paliar las posibles desigualdades generadas por el contexto familiar y proporcionar modelos y espacios alternativos que favorezcan actitudes de respeto y colaboración, partiendo en primer lugar de la construcción de la identidad personal de cada niño. La labor principal de la escuela, además de construir contextos y realidades basados en la igualdad, en la valoración positiva de la diversidad sin prejuicios ni discriminaciones, es ofrecer a los alumnos unos instrumentos y materiales que amplíen sus horizontes, lo impulsen a mirar con ojo crítico la realidad, desde diferentes ángulos y reflexionar acerca de los modelos impuestos por la sociedad.

Consideramos indiscutible el valor de la Literatura Infantil y Juvenil (LIJ) de cara al desarrollo integral de la persona y como herramienta educativa, de manera especial precisamente en referencia a la construcción de la identidad. Por lo tanto creemos necesario realizar un análisis de las implicaciones que la promoción de la igualdad de género ha 
conllevado en el ámbito de la LIJ, tanto desde el punto de vista editorial como creativo. De acuerdo con la temática que nos concierne podemos destacar, dentro del panorama literario actual, tres corrientes principales, en primer lugar aquella rama que sigue produciendo y promoviendo literatura para chicos y chicas, en segundo lugar la tendencia a crear intencionalmente obras dentro del marco de lo políticamente correcto y con una clara finalidad educativa, mientras que la tercera rama englobaría aquellas obras y autores auténticos, que escriben para los niños, al margen de los fines didácticos.

Autores como Roald Dahl, Astrid Lindgren o Margaret Mahy ofrecen al niño la posibilidad de asomarse al mundo desde otra óptica, desde una ventana situada a su altura pero que permite ver más allá del horizonte, y lo consiguen a través de un lenguaje, unas temáticas y unas formas libres de las constricciones propias de la edad adulta, ajustándose a las necesidades y formas de entender la vida propias de la infancia.

En el ámbito de la transmisión de principios de respeto y valoración del género y de la construcción de la identidad personal, nos parece especialmente interesante la labor de Bianca Pitzorno, una de las autoras contemporáneas más importantes en el panorama de la LIJ italiana, que ha creado y promovido una nueva escritura en femenino que se aleja de los estereotipos y de los moralismos fáciles, una escritora que simplemente narra el mundo (real e imaginario) desde el punto de vista de la niña que ha sido, como persona con todas sus peculiaridades, necesidades y sueños.

\section{Marco teórico}

Desde 1985 es obligatorio que todas las escuelas públicas o concertadas sean mixtas. Con la Ley Orgánica de Educación de 2006, tanto a nivel estatal como autonómico, se han regulado a nivel legislativo las actuaciones miradas a paliar las diferencias ligadas al género, tanto en términos de actitudes como de igualdad de oportunidades, con el fin de garantizar el avance hacia una sociedad futura más equilibrada y justa. En la LOE se resaltan entre los objetivos principales el desarrollo de la personalidad y capacidades afectivas de las personas, el respeto por los derechos fundamentales, la igualdad efectiva de oportunidades entre hombres y mujeres, el reconocimiento de la diversidad afectivo-sexual, así como la valoración crítica de las desigualdades que permita superar los comportamientos sexistas. En la Ley de Educación de 2006 se asume por lo tanto en su integridad el contenido de lo expresado en la Ley Orgánica 1/2004, de 28 de diciembre, de Medidas de Protección Integral contra la Violencia de Género. Las referencias en torno a tales conceptos se repiten en los apartados 
inherentes a los principios de la educación, los fines y en el desarrollo del currículo de todas las etapas educativas. Consecuentemente se han incrementado los programas y premios relacionados con la prevención de la violencia contra las mujeres y la coeducación (como los premios IRENE "La paz empieza en casa", la creación del grupo INTERCAMBIA "Educar en femenino y en masculino") y de forma paralela han cobrado fuerza e impulso asociaciones como el Instituto de la Mujer.

Todo individuo nace con una identidad sexual, biológica, en razón de la cual recibe un trato diferente y experimenta un proceso sistemático, más o menos estricto dependiendo del propio contexto, de tipificación sexual. El proceso de tipificación sexual, resultado de una construcción social de una imagen o modelo determinado de conductas, actitudes, cualidades o valores asignados a cada sexo, implica la aceptación y asimilación por parte del niño de una serie de expectativas y atribuciones relacionadas con su identidad sexual.

Amando López Valero y Carmelo Moreno Muñoz en La LIJ: su aportación a la conformación social de estereotipos sexistas (en Cano y Pérez, 2003) definen los estereotipos sexistas como modelos referidos a un grupo humano, basados en un juicio de valor, sentimiento o imagen que deben adaptarse a un modelo fijo y generalizado, no prestando atención a lo individual, y que pretenden legitimar la inferioridad o superioridad de un sexo respecto a otro. A pesar de que las diferencia individuales puedan explicarse con fundamento en relación al género en tan solo un 5\%, los estereotipos culturales se imponen con una fuerza tal que las personas llegamos a asumir como biológicas características y diversidades que son en realidad fruto del proceso de aprendizaje y socialización del individuo.

En el contexto escolar seguramente se han producido unos avances asombrosos en los últimos decenios en relación a la igualdad educativa y de oportunidades. No obstante, aun pudiendo hablar de superación de actitudes y episodios discriminatorios, a nivel de currículo oculto siguen estando presentes en buena medida expectativas y atribuciones diferenciadas en relación al género.

Por otra parte consideramos que, al igual que ocurre con la mayoría de las actuaciones relacionadas con valores o en general al margen de lo estrictamente académico, con demasiada frecuencia los proyectos relacionados con la construcción e igualdad de género quedan diluidos en el maremágnum de los aprendizajes formales. Además de traducirse en actividades puntuales y discontinuas, como la celebración del Día de la Mujer Trabajadora o Contra la Violencia de Género, la temática que nos concierne se aborda en nuestra opinión de 
una manera excesivamente directa y didáctica, que conduce más fácilmente al rechazo y negación que a la toma de conciencia, reflexión y apropiación de actitudes relacionadas con la igualdad de género.

En estos términos consideramos necesario en primer lugar adoptar un enfoque centrado en el desarrollo de la identidad de la persona a secas, partiendo de las peculiaridades y características personales propias de cada niño más allá del género. Directamente vinculado con ello reafirmamos el valor de la literatura como instrumento de desarrollo y construcción de la identidad, visto que ésta consiente abordar la realidad en un marco afectivo y lenguaje apropiados a la forma de entender el mundo propia del niño, librándolo (siempre que se lleve a cabo una selección adecuada) de moralismos gratuitos y simplistas.

La fuerza de la LIJ reside en que esta ofrece la posibilidad de ver con una mirada distinta la realidad a través del filtro de la metáfora, facilitando el proceso de toma de conciencia y reflexión acerca de sí mismo en un espacio seguro y sin juicios, de crear otros mundos posibles y personalizados.

\section{Panorama literario}

La literatura en general, y consecuentemente la LIJ, son reflejo del contexto ideológico histórico, cultural y político en el que se inscriben. La ideología se extiende en los textos literarios para expresar una concreta visión del mundo, tanto si se trata de obras que cuestionan como si apoyan al sistema imperante. Consecuentemente el panorama literario actual ha recogido y traducido los impulsos sociales de nuestra época.

En el ámbito de la construcción de la identidad de género, una rápida mirada hacia atrás nos ofrece incontables ejemplo del uso de la LIJ, sin reparo alguno, con fines moralizantes y adoctrinadores, como es el caso de las lecturas recomendadas para las jóvenes en España en los años '50. En un artículo de Mạ África Ibarra, publicado en la revista Bordón en 1949 (cit. Por Cerrillo y Sánchez, 2006), se destacan las diferentes necesidades lectoras de niños y niñas a partir de los 11/12 años, calificando de aptas para éstas últimas novelas de corte sentimental que desarrollen temas relativos a su femenina sensibilidad y ternura, enfocándolas en un recto sentido.

Tales recomendaciones nos llevan a la necesidad de remarcar que el análisis del panorama literario y su peso en términos educativo e ideológico, debe tener en cuenta no sólo la producción de la LIJ sino también su promoción y difusión. A modo de ejemplo podemos señalar que entre los textos se narrativa infantil de tradición oral, que se encuentran 
actualmente en el punto de mira de las críticas como manifestaciones de literatura sexista, encontramos un número considerable de cuentos en los que la mujer rompe con los estereotipos que la encasillan por convertirse en heroína independiente, astuta, valerosa... ${ }^{1}$ pero desde luego no son éstas las narraciones que mayormente se han difundido y promovido. Lo expuesto quiere poner en evidencia la responsabilidad compartida de autores, editores y personal docente en su rol de mediadores entre los niños y los libros, de cara a la selección de obras en el marco de la construcción de la identidad, con y sin género.

Sumariamente podríamos desglosar la situación literaria actual en lo referente a la LIJ y al género en tres corrientes principales: la primera, siguiendo la tradición, que ofrece obras directamente dirigidas a un público masculino o femenino, fuertemente estereotipada, no sólo por la presencia o protagonistas de uno u otro sexo, pero sobre todo en lo concerniente al mensaje subliminal transmitido por los paratextos. Baste con pensar en la colección GeronimoTea Stilton, la línea diseñada para niños emplea colores como el amarillo y el rojo, mientras que para las niñas se utilizan rosa, lila, purpurina, etc. Además hay que anotar que dentro del formato y características de la producción en términos literarios que no vamos a analizar o valorar aquí, la calidad de las obras de Tea resulta inferior, más simplona y edulcorada que los títulos dirigidos al público masculino. Otros ejemplos los constituyen las serie de Bat Pat y el Trío Beta, el Diario de Greg y de Niki o colecciones especialmente creadas para niñas como podrían ser Kika Superbruja, Witch, Monster High, Valeria Varita o las innumerables selecciones dedicadas a toda una galería de princesas rosa. Otra línea literaria es la que ha asumido directamente los postulados de la legislación y se dedica a la creación de textos políticamente correctos, con una clara intencionalidad educativa, presentando modelos alternativos, sobre todo por medio de la inversión de roles. El problema que surge en relación a tales producciones es que frecuentemente se trata de obras que se valen de un lenguaje, formato y narración artificiosos y forzados, excesivamente directas y a menudo creadas como respuesta a unos imperativos comerciales más que a los intereses y necesidades de los lectores. Finalmente la última línea de creación literaria está formada por aquellos autores que, sin más propósito o intencionalidad que narrar para el público infantil desde su propia experiencia y sentir, escriben con sinceridad y sin artificios. Es el caso de los clásicos de Astrid Lingren con Pippi Calzaslargas; de Karin Michaëlis y Bibi; de Burnett con Mary; de Roald Dahl

\footnotetext{
${ }^{1}$ Es el caso por ejemplo de: La niña sin brazos, La Baba Yaga, Caperucita Roja en la versión de Delarue.
} 
con Matilda, Sofía o la señorita Honey; de Christine Nöstlinger, que con la madre adoptiva de Konrad rompe por completo con muchos de los estereotipos femenino, de Margaret Mahy con Ernestina Laburnum o la inventora de Disparates y aventuras en el pico Huracán, de Henriette Bichonnier y su Lucila, de Babette Cole con su princesa Listilla o de Michael Ende con Momo. Todos los autores mencionados escriben al margen de cualquier estereotipo, sin constricciones, niñas y niños que luchan por hacerse sitio, por expresar sus ideas y su inconformidad con las injusticias del mundo. Se trata de literatura sin sexo, que habla de personas, de identidades diferentes más allá del género, que responden al imperativo de calidad literaria por el cual, según Kundera, el escritor debe ser capaz de suscitar preguntas y no ofrecer respuestas.

\section{Bianca Pitzorno}

"Bianca è esattamente quello che scrive, e scrive esattamente quello che è: una libertaria assoluta, cocciuta, struggente, irriverente, immersa fino al tallone, e oltre, in un'ironia umanistica e generosa, in complicità piena di vita e intelligenza, che i suoi lettori, istintivamente, riconoscono e amano." Piumini, R. ${ }^{2}$

En este marco consideramos que una atención especial la merece la autora Bianca Pitzorno, una de las más importantes autoras italianas contemporáneas en el ámbito de la LIJ, tanto por su labor como escritora, como colaboradora en el marco editorial y como icono de varias generaciones de lectores.

Para una lectura en profundidad de la obra de Bianca Pitzorno consideramos necesario trazar un breve diseño de la realidad italiana que enmarca los años de su formación como persona y escritora. La infancia y la adolescencia de la autora se sitúan entre el final de la Segunda Guerra Mundial, la postguerra y el milagro económico que vivió Italia al final de los años '50, todo ello "enriquecido" por los movimientos contestatarios de los años '60. La juventud de Pitzorno se desarrolla en la época del mito burgués en Italia, son los años del twist, de "La dolce vita" de Fellini, de la minifalda, de la beat generation, de los blue-jeans, de

\footnotetext{
2 Roberto Piumini, citado por Piumini Michaele en Pitzorno, Bianca, en La Società per l'Enciclopedia delle donne, Associazione Culturale, en: http://www.enciclopediadelledonne.it/biografie/bianca-pitzorno/: “Bianca es exactamente lo que escribe y escribe exactamente lo que es: una libertaria absoluta, tozuda, conmovedora, irreverente, comprometida hasta la médula, y más allá, en una ironía humanística y generosa, en complicidad llena de vida e inteligencia que sus lectores, instintivamente, reconocen y aman."(Traducción de la autora del presente artículo)
} 
la Barbie, del futbol y también de la Democracia Cristiana, del terrorismo, del feminismo, un contexto italiano pero no muy diferente de otras realidades europeas.

En 1946 se reconoce legalmente el derecho de voto para la mujer, en 1963 se promulga la ley 66 que permite a todas las mujeres acceder a cualquier carga y profesión, en 1962 se amplía la enseñanza obligatoria hasta los catorce años y en 1973 Elena Giannini Belotti publica "Dalla parte delle bambine" (Del lado de las niñas). En estos años el sistema educativo responde todavía a un modelo tradicional y rígido, niños y niñas están en clases separadas y en primaria la autora da sus primeros pasos en la escritura a la sombra de una maestra severa y cruel.

Bianca nace en una familia burguesa en una isla "aislada", donde escasean los recursos materiales, pero rodeada de un entorno cultural rico y estimulante. Sedienta de conocimiento, lectora compulsiva que cuenta entre sus obras favoritas con La Divina Comedia, Orlando furioso, La llíada y La Eneida y Horacio, a partir de los once años va al cine casi a diario. En sus años como estudiante de máster, frecuentando como oyente las clases de Arte Dramático en la Escuela de Recitación del Pequeño Teatro de Milán, es asidua espectadora de todas las obras teatrales ofrecidas a los estudiantes. Sus estudios arqueológicos se traducen en su obra en ese afán de precisión y rigor histórico que caracteriza sus narraciones, la experiencia con la televisión y la decepción provocada por la decadencia de la calidad cultural de los programas al final de los '70 determinan la presencia en su obra de una crítica hacia los medios de comunicación de masa. El amor hacia la mitología y la literatura latina llenan sus historias de guiños y referencias al mundo de la guerra de Troya y al Olimpo.

Bianca Pitzorno reconoce a Victor Hugo como uno de sus autores preferidos, por sus historias, pero también por su compromiso político y público, es abogada de la buena literatura sosteniendo que el objetivo literario y artístico debe prevalecer sobre lo pedagógico, que el escritor para niños debe mantener la misma disposición que el escritor para adultos, defensora del espíritu de libertad y de la importancia de los libros que hacen estremecerse, capaces de narrar la complejidad del mundo y mostrarnos más de nosotros.

La filosofía que subyace en la obra de la autora se basa en el derecho de libertad y de expresión de la personalidad de la infancia, es por ello que Bianca no habla a los niños, si no habla de niños o, mejor dicho, de niñas, dibujando, a través de su repleta galería de niñas 
recalcitrantes, una compleja historia de la infancia. A pesar de la marca femenina de su obra, la autora no se propone escribir novelas feministas, simplemente narra desde su vivencia y conocimiento del mundo. Esta idea se refleja en una entrevista para la revista de cultura cubana La Jiribilla:

“...me gusta escribir libros de aventuras, pero de la mente, del espíritu, de los sentimientos, escribo desde lo que sé. Soy una mujer, he sido una muchacha y una niña y lo que conozco es de eso. Si quiero contar a un ser humano desde dentro, pues tengo que hablar de las mujeres y las niñas. "

\section{La literatura de Bianca Pitzorno}

Probablemente lo más relevante con respecto a la literatura de Bianca Pitzorno sea su atención y dedicación a los problemas de la infancia en general, su concepción respetuosa de los mismos como dignos de ser representados y clamados en voz alta.

Las creaciones de Pitzorno constituyen una traducción literaria de los problemas relativos al ser mujer, chica o niña en la sociedad contemporánea, en un presente más o menos reciente. Sus protagonistas son niñas o adolescentes que ponen en relieve la hipocresía del mundo adulto, sus narraciones: reflexiones sobre problemas que van de la incapacidad de escuchar a los más pequeños a la indiferencia hacia los menos afortunados, la falta de tolerancia hacia la diversidad y la crítica a los medios de comunicación masiva o a las estructuras gubernativas, donde se equilibran de forma espontánea ponderación y diversión.

La casi totalidad de la producción de la autora es protagonizada por heroínas, enérgicas figuras femeninas que nada tienen que ver con el estereotipo de la niña buena y diligente, de las Barbies, todo aeróbica y vestiditos, si no que recuerdan y se inspiran en los modelos nórdicos de chica emancipada y sólida como Bibi o Pippi Calzaslargas. Tampoco podemos hablar de un retrato de la infancia despreocupada y serena, a pesar de no tratarse necesariamente de niños en situaciones desfavorecidas como la mayoría de los niños de Dahl, sino de una galería de chicas enfadadas, batalleras, decididas, intrépidas que se enfrentan con valor e inteligencia a los desequilibrios y a las injusticias que las rodean. En efecto, independientemente de la presencia de adultos más o menos atentos, las protagonistas de Bianca Pitzorno, con su tierna edad, muestran constantemente su autonomía y capacidad de pensar por sí mismas, dueñas de sus acciones sin dejarse influenciar por las expectativas 
externas, seguras en sus elecciones y decisiones. En su literatura rompe con el estereotipo de niños que infringen las reglas, inconformista y de niñas que actúan dentro del marco de lo permitido, por medio de un desfile variopinto de chicas aventureras con las ideas claras, a menudo apodadas "marimachos" pero que no dejan por ello de mantenerse fieles a sus principios y formas de ser y estar.

Así mismo, cabe destacar la peculiaridad de los nombres que la autora selecciona cuidadosamente para sus heroínas, herencia de la pasión despertada en la autora por los fascinantes nombres bíblicos, que frecuentemente hacen alusión a la mitología latina y griega, sobre todo teniendo en cuenta que la misma escritora afirma que el nombre es el primer signo distintivo de una persona. Prisca, Làlage, Lavinia, Aglaia, Rosalba desde la primera página, a través del nombre la autora dibuja unos personajes con espesor y carácter, otorgándoles fuerza y personalidad, reforzando la construcción de una personalidad única e irrepetible.

\section{La obra}

Extraterrestre en igualdad, una novela para reflexionar acerca de la diferencia de género de forma original y divertida, es probablemente la más representativa del compromiso de la autora con lo femenino y con la identidad. Por medio de un programa de intercambio interestelar, Mo, un extraterrestre proveniente del planeta Deneb, llega a la Tierra para pasar una temporada con una familia italiana. Mo no sabe a qué sexo pertenece, en su planeta esta información es irrelevante, cuando nace una nueva criatura se mira si está sana, no si es varón o hembra. Los niños al nacer no presentan diferencias, hasta los 50 años (20 años terrestres) no se manifiestan los atributos sexuales y no se conoce el propio sexo, por otra parte necesario sólo para la reproducción y no para la elección de profesión, intereses, amigos o actitudes. Pero en la Tierra conocer el sexo de un individuo es imprescindible para su educación, desde la elección de la ropa al corte de pelo, desde el colegio a las amistades. Mo tendrá que aceptar cargar con las medidas resolutivas aplicadas por la sociedad terrestre, sin llegar a comprenderlas, sintiéndose en todo momento demasiado encorsetado por los roles que le son impuestos. Mo descubrirá que toda la importancia que en la Tierra se otorga al sexo es un modo para coartar las libertades, trazar, desde el momento del nacimiento, límites estrictos y claustrofóbicos.

La obra es una denuncia a la discriminación y prejuicios de género que complican la vida para ambas parte, en efecto, aunque ponga evidentemente en relieve las desventajas de 
ser mujer ("Estoy muy contenta de haber nacido en Deneb, ya que tenía que nacer mujer" dice Mo), la autora no deja de poner en evidencia como los privilegios masculinos tienen un precio muy alto:

"iAhora he comprendido porque para los terrestres es tan importante saber en seguida si el recién nacido es varón o hembra! Está claro: si no lo entrenan un tiempo suficientemente largo, ninguna de sus hijas, en el momento adecuado, tendrá la suficiente paciencia para ser capaz de hacer de mujer."

"Y no te creas que a ser varón se gana mucho (...) Se tienen muchos privilegios pero se pagan caros. (...) No puede conmoverse, no puede tener miedo, no puede ser sensible, no puede ser tierno y amable, no puede llorar en público, no puede cantar y bailar, no puede ponerse vestidos bonitos, no puede elegir ocuparse de la casa y de los niños en lugar de ir a la oficina..."

Los diferentes personajes femeninos que rodean a Mo representan las diferentes maneras de ser mujer, eligiendo someterse a las imposiciones sociales, encorsetarse para encajar en la comunidad de pertenencia, o rebelarse, escandalizando o desde la sombra, a los imperativos sociales, reivindicando el derecho a respetar la propia naturaleza.

A través de una historia original y de una narrativa ágil, que combina a la perfección diversión y profundidad, por medio de un lenguaje rico y cuidado (remarcamos que en las cartas que MO envía a su planeta se omiten por completo las desinencias de género) Bianca Pitzorno hace luz sobre los aspectos más evidentes de la influencia y peso de la educación sobre el carácter, gustos y actitudes de las personas. Al mismo tiempo pone de relieve como los intereses y formas de ser y sentir personales son mucho más relevantes que el género que nos es asignado por la naturaleza. Sin moralismo alguno la autora estimula la reflexión sobre la presión, injusta aun cuando ejercida con las mejores intenciones, que recibimos del entorno con respecto a la identidad de género y a la formación de una personalidad socialmente aceptable.

\section{Las chicas liberadas}

A parte de la obra Extraterrestres en igualdad Bianca Pitzorno nos brinda una larga lista de modelos femeninos liberados, que rompen con todo estereotipo y prejuicio. La

\footnotetext{
${ }^{3}$ La citación se inspira en un pensamiento de Jean Jaques Rousseau: "Las mujeres deben ser educadas desde el principio a llevar el yugo, de forma de no resentir demasiado por su peso".
} 
creación destinada a los lectores más jóvenes (entre los siete y los diez años) podría calificarse de "unisex", independientemente de la presencia constante de protagonistas femeninas.

La casa en el árbol, historia de una mujer y una niña sin vínculos parentales que deciden libremente vivir en un árbol, es una oda a la libertad que ofrece una visión totalmente revolucionaria de las jerarquías humanas. Nuevamente la autora subvierte los lugares comunes presentando una "familia" sin retórica ni tiernos chantajes, compuesta por dos personas que se han gustado y eligen estar juntas, donde cada uno encuentra su sitio, su canal de expresión y asume sus responsabilidades de manera directamente relacionada con la dignidad humana más allá del estatus social. Bianca y Aglaia, las dos protagonistas, vuelven a convertirse en un modelo femenino que supera el discurso del género, que no se puede encasillar pero tampoco pierde su idiosincrasia a favor de las características propias del otro sexo. El don de Bianca es su capacidad de dibujar identidades, personalidades únicas, con sus cualidades y defectos, y precisamente por ello tan atractivas y perfectas.

Con Pequeña bruja, gracias a la ironía y espontaneidad que la caracterizan, Bianca Pitzorno juega inteligentemente, a caballo de la escoba de su feminismo rugiente, con la figura de la bruja y sus dobles atribuciones: mujer perseguida y mujer con poderes extraordinarios. Siempre en un contexto eminentemente femenino, una familia de siete hermanas que por circunstancias laborales de los padres tienen que desenvolverse autónomamente ${ }^{4}$, y sobre la base de una fundamentación científica cuidadosa en torno a las características identificativas de una bruja, la escritora nos presenta una pequeña hechicera que consigue con sus encantos arrancar por completo todo prejuicio asociado a la figura de la mujer-bruja. El personaje de Emilia, la pequeña bruja, se convierte en una metáfora de la excepcionalidad de la infancia, que consigue conjugar la realidad con lo extraordinario. Por lo tanto volvemos a enfrentarnos a un modelo femenino que, a pesar de su peculiaridad y su ser y sentir como niña, transciende los límites del género, desde la esencia de una identidad personal.

De manera similar en el concepto mencionamos las dos magníficas novelas Escúchame el corazón, crónica de una año escolar que recalca en clave femenina Corazón de De Amicis pero librándose de la moral conmovedora y de la óptica adulta de éste, y Diana, Cupido y el comendador, relato de las vicisitudes de una niña entre infancia y adolescencia y de

\footnotetext{
4 Destacamos como recurrente en la obra de la autora la creación de sistemas sociales y familiares basados en la equidad y en la libertad que invierten las jerarquías de autoridad arbitraria, en los que la figura del adulto frecuentemente desaparece.
} 
su lucha contra las hipocresías e incoherencias del mundo adulto. En ambas novelas Bianca Pitzorno propone unas heroínas excepcionales en su normalidad, luchadoras y anticonformistas, con todos sus sentimientos, incertidumbres, frustraciones, alegrías...coherentes e independientes, reacias a dejarse guiar por unos imperativos externos y en desacuerdo con su forma de entender la realidad. Prisca y Diana son fieles a sí mismas, escuchan los latidos e impulsos de su corazón y por ello se convierten en únicas entre miles de niñas que se parecen.

\section{Crecer en femenino}

A parte de su inmejorable labor como escritora, debemos reconocer el mérito de Bianca Pitzorno en relación a su compromiso con la construcción de identidades y con el ser mujer, como co-creadora de la colección editada por Mondadori "Gaia Junior", colección destinada a ofrecer a las adolescentes una escritura en femenino pero seria y comprometida, rehuyendo del formato de la novelita rosa, pero reconociendo las exigencias e inquietudes que caracterizan esta etapa en la vida de una mujer.

Negar la existencia de unas peculiaridades y diferencias en la forma de ser y de sentir ligadas al género sería negar la existencias de dos sexos y podría inducir a caer en el error, en aras de demostrar la igualdad de género, de legitimar la supremacía de las cualidades propiamente masculinas, anulando y menospreciando las propias del sexo femenino. Además cabe considerar que en la actualidad aún estamos lejos de superar por completo la transmisión cultural y social de modelos ligados al género, por lo que se vuelve fundamental ofrecer una respuesta de calidad, literaria y existencial, a las necesidades existentes independientemente de su origen más social que biológico. Por otra parte la adolescencia es uno de los momentos más sensibles y cruciales en la formación de la identidad, en el que las niñas (y los niños) buscan y necesitan modelos para su devenir adulto.

Las novelas de Bianca Pitzorno destinadas a la adolescencia se alejan totalmente del tono maternal, de los diminutivos tiernos, de la narración con tintas condescendientes, de los clichés que caracterizan las novelas rosa que describen supuestas pequeñas contrariedades de las chicas que acaban resolviéndose felizmente gracias al sacrificio y a la bondad. La autora aborda temas propios de la adolescencia como los anhelos, la admiración frustrada, la mitificación, la amistad, la individualidad, el peso del cuerpo y de la imagen, el alma, la muerte, mostrando una gran capacidad por dar vida con sus palabras y narraciones al frágil equilibrio y 
a la fuerza de las emociones e inquietudes vitales que constituyen la esencia misma de la adolescencia.

Entre las obras publicadas por la autora destinadas al público adolescente queremos destacar Speciale violante, Re mida ha le orecchie d'asino y Principessa Laurentina. Las protagonistas de estas obras son chicas normales con grandes sueños, simpáticas, no superficiales pero aun "indefinidas", con sus debilidades y su capacidad para levantarse y seguir su camino tras la derrota.

Con su característico tono sutilmente crítico y humorístico, cargado de significados y emociones, en Speciale Violante Bianca narra la historia de tres amigas que tienen que enfrentarse al impacto que causa la llegada de una troupe televisiva al pequeño pueblo en el que veranean, a la dificultad por separar ficción y realidad, mitificación y mirada crítica. Entre las líneas de la narración se debaten los anhelos, las decepciones, los conflictos, las certezas y las inseguridades de tres adolescentes cuya sólida y sincera amistad se ha construido sobre la base de tres personalidades que se completan en su individualidad. La autora traduce maravillosamente la adolescencia en un momento de ser, de soñar a lo grande, en el reflejo interno de lo cotidiano, a mano de unas protagonistas aun sin espesor, pero que dejan entrever unas personalidades fieles a unos valores y principios sólidos y seguros.

Quizás Re Mida ha le orecchie d'asino sea la muestra más evidente de la capacidad de la escritora por captar la sutil línea de tránsito entre infancia y adolescencia. La profunda amistad que une a Làlage e Irene, ambas de 11 años, es puesta en peligro por el afán frustrado de la primera por agradar a la fascinante prima dos años mayor que ella. En un verano enredado de secretos y mentiras, compañías de teatro giróvagas, enamoramientos reales y fantaseados, la protagonista se debate entre la amistad sincera y la admiración sumisa, entre el amor y la muerte, la corporeidad y la espiritualidad, lo interior y exterior del cuerpo. Con una narrativa cercana y profunda, sin maquillajes ni tabúes, Bianca Pitzorno ofrece una reflexión sobre la materialidad, el peso la corporeidad y su rol en la adquisición de la experiencia, sobre la fragilidad del cuerpo y la (posible) inmortalidad del alma, sobre la individualidad y la autodefinición. Y nos regala Làlage, nuevamente más allá de lo esperable, quieta y reflexiva pero llena de pulsiones y preguntas, extraordinaria y perfecta en sus contradicciones, en su pausada e impetuosa lucha por crecer. 
Por último en Principessa Laurentina Pitzorno narra la historia de Barbara, una adolescente inquieta y rebelada frente a los cambios, cambios materiales relacionados con la separación de sus padres, su traslado a otra ciudad que la separa de sus amistades y referencias, la llegada de una hermanastra que la desplaza y ensombrece y cambios existenciales, la lucha con un cuerpo que se transforma y que no reconoce, la búsqueda de nuevos afectos y de su sitio en el mundo. La visión rabiosa de una adolescente que se siente incomprendida por sus padres, traicionada por Liselotte, la niñera, en realidad instrumento de la madre para llegar a conocer las intimidades y sentimientos de su hija, pero también la visión preocupada e impotente de una madre que no consigue comunicar con su hija. Es un desenlace trágico el que permite que Barbara florezca, que cruce el umbral de la madurez y vaya progresivamente encontrando su lugar y a sí misma. Una novela sobre el conflicto entre padres y adolescentes, sobre la dificultad de comprenderse mutuamente, sobre la aceptación y las dificultades del hacerse adulto, sobre una adolescencia marcada por los sentimientos de frustración e inadecuación. Pero también una novela sobre la pérdida y el reencuentro, sobre la canalización de las pulsiones. De nuevo la autora habla sin adornos ni edulcorantes de temáticas tan trascendentes como la muerte y el remordimiento, la búsqueda de la propia identidad, la frustración, el cuerpo y el espíritu, de nuevo nos regala una imagen intensa y auténtica del ser femenino.

En una actualidad en la que el devenir mujer ha perdido sus puntos de referencia, en la que imperan aun unas expectativas y atributos sociales poderosos con respecto a la identidad femenina pero al mismo tiempo se ha vuelto casi necesario demostrar constantemente la capacidad de actuar y desenvolverse como hombres, en la que crecer como mujer significa debatirse entre tradición y emancipación, muchas veces entendida como homogeneización a los modelos masculinos, la literatura de Bianca Pitzorno ofrece unos modelos que trascienden la identidad de género para dar respuesta a las reales exigencias de niños y jóvenes en lo concerniente a la autodefinición y expresión libre de su ser y estar.

Bianca Pitzorno es una de aquellas narradoras capaces de sorprender y enamorar a su público, infantil y adolescente, pero también adulto, gracias a su maravillosa habilidad por 
combinar las palabras de tal forma que lleguen a conectar con lo más íntimo y personal de cada uno.

Consideramos que su calidad literaria, su atrevimiento conceptual, su humor y la profundidad de su producción la convierten en uno de los pilares de la LIJ a nivel universal y, sobre todo, puede ayudarnos a tomar conciencia, aceptar y valorar nuestra identidad única, posibilitando el "atrevimiento" de ser persona más allá de un género.

\section{Referencias Bibliográficas}

CANO VELA, A. Y PÉREZ VALVERDE, C. (coord.) (2003): Canon, literatura infantil y juvenil y otras literaturas. Cuenca: Ediciones de la Universidad de castilla la Mancha CERRILLO, P. y SÁNCHEZ, C. Literatura con mayúsculas. En Ocnos 2006, 2, pág. 7-21 HORMILLA, H.(2011) : Escribir sin hipocresía, en Lahiribilla, año X, 3-9 de diciembre, La Habana. Disponible en: http://www.lajiribilla.cu/2011/n552_12/552_24.html. Consultado el 05/05/2014 PITZORNO, BIANCA (1994): Diana, Cupido e il commendatore. Milano: Mondadori PITZORNO, B. (2008): Escúchame el corazón. Madrid: Sabina Editorial PITZORNO, B. (1991): Extraterrestri alla pari. Torino: Einaudi PITZORNO, B. (2005): La casa en el árbol. Madrid: Anaya PITZORNO, B. (2009): Pequeña Bruja. Sevilla: Kalandraka PITZORNO, B. (1997): Principessa Laurentina. Milano: Gaia Junior Mondadori PITZORNO, B. (2012): Re Mida ha le orecchie d'asino. Milano: Mondadori PITZORNO, B. (1998): Speciale violante. Milano: Gaia Junior Mondadori PIUMINI, M.: BIANCA PIOZORNO, La Società per l'Enciclopedia delle donne Associazione Culturale, Consultado el 05/05/2014, disponible en: http://www.enciclopediadelledonne.it/biografie/bianca-pitzorno/

\section{Referencias Legislativas}

España, Ley Orgánica 2/2006, de 3 de mayo, de Educación

España, Ley Orgánica 1/2004, de 28 de diciembre, de Medidas de Protección Integral contra la Violencia de Género 